\title{
COMPATIBLE COARSE NODAL AND EDGE ELEMENTS THROUGH ENERGY FUNCTIONALS
}

\author{
F. MUSY $^{\dagger}$, L. NICOLAS ${ }^{\ddagger}$, AND R. PERRUSSEL ${ }^{\dagger \ddagger}$
}

\begin{abstract}
We propose new algorithms for the setup phase of algebraic multigrid (AMG) solvers for linear systems coming from edge element discretization. The construction of coarse levels is performed by solving an optimization problem with a Lagrange multiplier method: we minimize the energy of coarse bases under a constraint linking coarse nodal and edge element bases. On structured meshes, the resulting AMG method and the geometric multigrid method behave similarly as preconditioner. On unstructured meshes, the convergence rate of our method compares favorably with the AMG method of Reitzinger and Schöberl.
\end{abstract}

Key words. multigrid, constrained optimization, edge finite elements

AMS subject classifications. 65F10, 65N55, 65N30

1. Introduction. Edge element discretization plays a key-rôle in computational electromagnetism; it can be implemented for evaluating the electric or magnetic field using the vector wave equation or for evaluating the magnetic potential using the eddy current formulation. As this finite element discretization leads to sparse but generally large linear systems, efficient methods are required for the solution. Multilevel techniques have been introduced by Hiptmair [5] and Arnold et al. [1]; they are shown to be optimal on a hierarchy of nested grids. However, the inherent need of hierarchical finite element meshes in geometric multigrid methods is very restricting for industrial applications. It is more convenient to make use of a grey-box multilevel algorithm which needs a single grid but takes into account additional information on the initial problem.

For this purpose, algebraic multilevel methods have already been developed. Beyond the use of specific smoothers as Hiptmair or Arnold et al. proposed, Reitzinger and Schöberl 14 have highlighted an essential geometric compatibility relation which means that the gradient of a coarse nodal element function must belong to the coarse edge element space. Based on this relation, Reitzinger and Schöberl achieved an efficient method which is now widespread in the computational electromagnetism community 17, 9, 10, 18, 17. However, the combinatorial approach used there does not lead to an optimal convergence rate. Some improvements have been proposed by Bochev et al. [2, 6] based on smoothed aggregation techniques [15] and a compatibility with a larger class of nodal prolongation operators.

Here, we propose a somewhat different approach. Following ideas from 16 and [19], we construct coupled nodal and edge coarse bases by energy minimization and we enforce the compatibility relation as a constraint. A connection can be made with the smoothed aggregation method [8], which also involves energy minimization. In order to simplify the notations, we present our construction in a two-level framework.

In Section 2, the problem and the properties of the considered finite element space are reviewed, especially the essential geometric relation. The constrained minimization problem is presented in Section 3. Then we introduce the resulting linear system with Lagrange multipliers and the edge prolongation matrix as unknowns. Section 1

\footnotetext{
${ }^{\dagger}$ CNRS, UMR5208, Institut Camille Jordan, Villeurbanne, F-69622, France; Ecole Centrale de Lyon, Ecully, F-69134, France; université de Lyon, Lyon, F-69003, France.

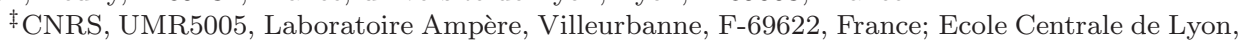
Ecully, F-69134, France; université de Lyon, Lyon, F-69003, France.
} 
describes in detail the conditions to obtain a well-posed minimization problem. First, we clarify the construction of the edge function supports from a given graph and the coarse nodal function supports and we point out the satisfied constraints. Then, we prove that the coarse graph has to satisfy a connectivity condition if the implicit constraints are not enforced and we propose a construction of the coarse nodal function supports together with a suitable definition of the coarse graph. Finally in Section 5 , we evaluate the efficacy of various versions of the algorithm corresponding to different choices of energy norm. We give numerical results for $2 \mathrm{D}$ and $3 \mathrm{D}$ problems on structured and unstructured meshes. On a hierarchy of nested meshes, the edge bases being computed from the geometric nodal bases at each level, the resulting AMG method and the geometric multigrid method behave similarly as preconditioner. On unstructured meshes, the convergence rate of our method is better than the method of Reitzinger and Schöberl 14. However, because of the solution of the constrained minimization problem, we pay this improvement with a large increase of the computing cost for the edge bases.

\section{Definition of the continuous problem and its discretization.}

2.1. Formulation. The following problem has to be solved on a domain $\Omega \subset \mathbb{R}^{d}$, $d=2,3$ :

$$
\left\{\begin{array}{l}
\text { To find } \mathbf{E} \in V \text { such that: } a\left(\mathbf{E}, \mathbf{E}^{\prime}\right)=F\left(\mathbf{E}^{\prime}\right), \forall \mathbf{E}^{\prime} \in V_{0}, \\
\text { with } a\left(\mathbf{E}, \mathbf{E}^{\prime}\right)=\int_{\Omega} \delta \operatorname{curl} \mathbf{E} \cdot \operatorname{curl} \mathbf{E}^{\prime}+\int_{\Omega} \gamma \mathbf{E} \cdot \mathbf{E}^{\prime}
\end{array}\right.
$$

$V$ is an affine subspace of $\mathbf{H}(\operatorname{curl}, \Omega)$ 11 taking into account essential boundary conditions, $V_{0}$ is the vector subspace parallel to $V, F$ is a linear form on $V_{0}$ which describes the source term, $\delta$ and $\gamma$ are strictly positive functions, so that $a$ is coercive; $F, \delta$ and $\gamma$ depend on the applications under consideration.

This formulation includes many static and transient electromagnetic models: potential vector formulation for magnetostatic or eddy currents, electric or magnetic field formulation in the transient case. For instance, for an eddy current formulation in the transient case discretized by an implicit Euler scheme with time parameter $\triangle t$, $\mathbf{E}$ is the electric field, $\delta$ is equal to $\Delta t / \mu$ with $\mu$ the magnetic permeability and $\gamma$ is the electric conductivity.

2.2. Finite element space and properties. Problem 2.1 is discretized by using the lowest order edge elements introduced by Nédélec 13 , which are conforming in $\mathbf{H}($ curl, $\Omega$ ). We consider triangular and tetrahedral meshes. For a tetrahedron $K$, the local polynomial space $V_{K}$ is defined by:

$$
V_{K}=\left\{x \mapsto \mathbf{p} \times \mathbf{x}+\mathbf{q}, \mathbf{x} \in K \text { and } \mathbf{p}, \mathbf{q} \in \mathbb{R}^{3}\right\} .
$$

The symbol $\times$ denotes the cross-product.

The local degrees of freedom which permit to ensure the conformity in $\mathbf{H}(\operatorname{curl}, \Omega)$ are given by path integrals along the edges of the element:

$$
\mathbf{E}_{h} \mapsto \int_{e} \mathbf{E}_{h} \cdot \mathbf{t} \mathrm{ds}
$$

where $e$ is an edge of $K, \mathbf{t}$ is a tangential vector to $e$ and $\mathbf{E}_{h}$ belongs to $V_{K}$. Based on this local description, an edge finite element space can be defined on a mesh of the 
domain. $V_{h}$ will denote the edge finite element space, defined on a mesh $T_{h}$ of the domain $\Omega$, and taking into account essential boundary conditions.

Therefore using this finite element space, we are led to solve a linear system:

$$
A x=f .
$$

A fundamental property links the edge elements to the nodal $P_{1}$-Lagrange elements: the gradient of a nodal function belongs to the space of edge functions. If we denote the finite element bases as follows:

(i) $\left(\phi_{p}^{h}\right)_{p=1, \ldots, N^{h}}$ is the nodal basis,

(ii) $\left(\lambda_{i}^{h}\right)_{i=1, \ldots, E^{h}}$ is the edge basis,

this property can be recast as:

$$
\operatorname{grad} \phi_{p}^{h}=\sum_{i=1}^{E^{h}} G_{i p}^{h} \lambda_{i}^{h}, \forall p \in\left\{1, \ldots, N^{h}\right\},
$$

where $G^{h}$ is known and is the edge-node incidence matrix of the mesh $T_{h}$; in what follows, the superscript $h$ for the fine level will be opposed to $H$ used for the coarse level. $G^{h}$ is a discrete analogue of the gradient operator on this mesh. Moreover, if the domain is contractible, the subspace spanned by the $\operatorname{grad} \phi_{p}^{h}$ 's coincides exactly with the kernel of the curl operator in the edge element space [⿶.

Our aim, as highlighted in [14], is to preserve the analogous compatibility relation for coarse bases, which is natural with a hierarchy of nested grids. Thus, a coherent representation of the kernel of the curl operator is preserved, which permits the efficient use of the smoothers proposed by Hiptmair [5] and by Arnold et al. [1]. Therefore the gradient of a coarse nodal function must be a combination of coarse edge functions.

Let us introduce the following notations:

(i) $\left(\phi_{n}^{H}\right)_{n=1, \ldots, N^{H}}$ is the coarse nodal basis,

(ii) $\left(\lambda_{e}^{H}\right)_{e=1, \ldots, E^{H}}$ is the coarse edge basis,

the compatibility condition is written algebraically as:

$$
\operatorname{grad} \phi_{n}^{H}=\sum_{e=1}^{E^{H}} G_{e n}^{H} \lambda_{e}^{H}, \forall n \in\left\{1, \ldots, N^{H}\right\} .
$$

where $G^{H}$ is the discrete analogue of the gradient operator and is the edge-node incidence matrix of a coarse graph. This operator $G^{H}$ or equivalently the associated coarse graph has to be constructed before the computation of the coarse edge basis. The way to obtain the coarse graph from the fine graph is postponed to Section 1 .

These coarse bases are constructed so as to satisfy the inclusion of finite element spaces, the "coarse" being included in the "fine", which is expressed by the following algebraic relations:

$$
\begin{aligned}
& \phi_{n}^{H}=\sum_{p=1}^{N^{h}} \alpha_{p n} \phi_{p}^{h}, \forall n \in\left\{1, \ldots, N^{H}\right\}, \\
& \lambda_{e}^{H}=\sum_{i=1}^{E^{h}} \beta_{i e} \lambda_{i}^{h}, \forall e \in\left\{1, \ldots, E^{H}\right\} .
\end{aligned}
$$


According to relations (2.5), 2.6) and (2.7), the components $\alpha_{p n}$ and $\beta_{i e}$ of the coarse bases must satisfy:

$$
\sum_{e=1}^{E^{H}} \sum_{i=1}^{E^{h}} \beta_{i e} G_{e n}^{H} \lambda_{i}^{h}=\sum_{p=1}^{N^{h}} \sum_{i=1}^{E^{h}} G_{i p}^{h} \alpha_{p n} \lambda_{i}^{h}, \forall n \in\left\{1, \ldots, N^{H}\right\},
$$

or equivalently:

$$
\sum_{e=1}^{E^{H}} \beta_{i e} G_{e n}^{H}=\sum_{p=1}^{N^{h}} G_{i p}^{h} \alpha_{p n}, \forall i \in\left\{1, \ldots, E^{h}\right\}, \forall n \in\left\{1, \ldots, N^{H}\right\} .
$$

Finally, if $\alpha$ denotes the matrix of components $\alpha_{i n}$ and $\beta$ the matrix of components $\beta_{i e}$, the compatibility relation (2.6) writes also under assumptions (2.5) and (2.7) as:

$$
\beta G^{H}=G^{h} \alpha .
$$

\section{Overview of the coarse bases construction.}

3.1. Energy minimization problems. The domain $\Omega$ is decomposed into overlapping subdomains $\Omega_{n}^{H}$, for $n$ in $\left\{1, \ldots, N^{H}\right\}$; this decomposition enables us to restrict the support of the coarse nodal basis functions.

In the same way, $\Omega$ is decomposed into overlapping subdomains $\mathcal{U}_{e}$, for $e$ in $\left\{1, \ldots, E^{H}\right\}$ in order to localize the supports of the coarse edge basis functions. The subdomain $\mathcal{U}_{e}$ will be the intersection of two subdomains $\Omega_{l}^{H}$ and $\Omega_{m}^{H}$.

Given the subdomains $\Omega_{n}^{H}$, the choice of the matrix $G^{H}$ determines the definition of the subdomains $\mathcal{U}_{e}$; we will clarify this point in Subsection 1.1 .

Two minimization problems under constraints are solved successively. In analogy with the nodal element case 16], we first solve:

$$
\left\{\begin{array}{l}
\text { To find }\left(\phi_{n}^{H}\right)_{n=1 . . N^{H}} \text { minimizing } \sum_{n=1}^{N^{H}} c\left(\phi_{n}^{H}, \phi_{n}^{H}\right) \text { under the constraints: } \\
\sum_{n=1}^{N^{H}} \phi_{n}^{H}(x)=1, \forall x \in \bar{\Omega} \text { and } \operatorname{supp}\left(\phi_{n}^{H}\right) \subset \overline{\Omega_{n}^{H}}, \forall n \in\left\{1, \ldots, N^{H}\right\}
\end{array}\right.
$$

The bilinear form $c$ can be for instance $c(\phi, \psi)=\int_{\Omega} \gamma \operatorname{grad} \phi \cdot \operatorname{grad} \psi$ or more simply the bilinear form associated with the Laplacian of the graph i.e. $\left(G^{h}\right)^{t} G^{h}$. Here the superscript $t$ denotes transposition.

Next, from the coarse nodal basis $\left(\phi_{n}^{H}\right)_{n=1, \ldots, N^{H}}$ we compute the coarse edge basis $\left(\lambda_{e}^{H}\right)_{e=1, \ldots, E^{H}}$ by solving the problem:

$$
\left\{\begin{array}{l}
\text { To find }\left(\lambda_{e}^{H}\right)_{e=1, \ldots, E^{H}} \text { minimizing } \sum_{e=1}^{E^{H}} b\left(\lambda_{e}^{H}, \lambda_{e}^{H}\right) \text { under the constraints: } \\
\operatorname{grad} \phi_{n}^{H}=\sum_{e=1}^{E^{H}} \lambda_{e}^{H} G_{e n}^{H}, \forall n \in\left\{1, \ldots, N^{H}\right\} \text { and } \operatorname{supp}\left(\lambda_{e}^{H}\right) \subset \overline{\mathcal{U}_{e}}, \forall e \in\left\{1, \ldots, E^{H}\right\} .
\end{array}\right.
$$


The letter $b$ denotes a scalar product on $V_{h}$ which can be the bilinear form $a(\cdot, \cdot)$ from (2.1) or variants, which will be introduced in Subsection 5.2 and implemented in numerical examples in Section 5 .

Problem (3.1) can be solved by using the method described in [16]. This is the reason why we mainly concentrate our attention on Problem (3.2).

We introduce algebraic notations which encode the support constraints. First for all $e$ in $\left\{1, \ldots, E^{H}\right\}, I_{e}$ is a subset of the set of indices of the fine edge basis functions whose support is included in $\overline{\mathcal{U}_{e}}$. We assume that for all $e I_{e}$ is non empty and we define:

$$
\widetilde{M}=\sum_{e=1}^{E^{h}}\left|I_{e}\right| .
$$

The term $|Z|$ denotes the number of elements in a finite set $Z$. Then for $e$ in $\left\{1, \ldots, E^{H}\right\}$ with $I_{e}=\left\{i_{1}, \ldots, i_{\left|I_{e}\right|}\right\}$, we introduce the projection operator which keeps only the components indexed by $I_{e}$ :

$$
\begin{aligned}
& Q_{e}: \mathbb{R}^{E^{h}} \rightarrow \mathbb{R}^{\left|I_{e}\right|}, \\
& \left(Q_{e} x\right)_{k}=x_{i_{k}}, \forall x \in \mathbb{R}^{E^{h}}, \forall k \in\left\{1, \ldots,\left|I_{e}\right|\right\} .
\end{aligned}
$$

The term $x_{i_{k}}$ denotes the $i_{k}$-th component of $x$. It is straightforward that the transposed operation $Q_{e}^{t}$ is defined by :

$$
\begin{aligned}
& Q_{e}^{t}: \mathbb{R}^{\left|I_{e}\right|} \rightarrow \mathbb{R}^{E^{h}} \\
& \left(Q_{e}^{t} y\right)_{i}=\left\{\begin{array}{l}
0 \text { if } i \notin I_{e} \\
y_{k} \text { if } i=i_{k} \in I_{e}
\end{array}, \forall y \in \mathbb{R}^{\left|I_{e}\right|} .\right.
\end{aligned}
$$

Secondly, in order to restrict the number of constraints in Problem (3.2), we introduce sets $J_{n}$ for all $n$ in $\left\{1, \ldots, N^{H}\right\}$. The sets $J_{n}$, like the sets $I_{e}$, are subsets of indices of the fine edge basis functions.

The sets $J_{n}$ will be carefully defined with the help of support conditions, so as to decrease as much as possible the number of constraints coming from (2.8b) and to obtain a well-posed problem; the construction of the $J_{n}$ 's is described in Subsection 4.2. We introduce an integer for denoting the number of constraints :

$$
M=\sum_{n=1}^{N^{H}}\left|J_{n}\right| .
$$

Since some $J_{n}$ 's can be empty, we introduce the set:

$$
F=\left\{n \in\left\{1, \ldots, N^{H}\right\} \mid J_{n} \neq \emptyset\right\} .
$$

Similarly to the operator $Q_{e}$ from set $I_{e}$, we introduce a canonical projection operator associated to $J_{n}$ for $n$ in $F$ :

$$
R_{n}: \mathbb{R}^{E^{h}} \rightarrow \mathbb{R}^{\left|J_{n}\right|}
$$

Let $K$ be the matrix whose coefficients are $b\left(\lambda_{j}^{h}, \lambda_{i}^{h}\right)$. In order to obtain a matrix form of the minimization problem, we define matrices which are restrictions to the subdomains under consideration of the matrix $K$ :

$$
K_{e}=Q_{e} K Q_{e}^{t}
$$


We also define:

$$
\xi_{n}=R_{n} G^{h} \alpha_{\bullet n}, \forall n \in F \text { and } \beta_{e}=Q_{e} \beta_{\bullet e}, \forall e \in\left\{1, \ldots, E^{H}\right\} .
$$

where $\alpha_{\bullet}$ denotes the $n$-th column of $\alpha$ and $\beta_{\bullet}$ the $e$-th column of $\beta$.

After solving Problem (3.1), which gives the matrix $\alpha$, we compute the $\beta_{e}$ 's by solving the problem:

$$
\left\{\begin{array}{l}
\text { To minimize } \sum_{e=1}^{E^{H}} \beta_{e}^{t} K_{e} \beta_{e} \text { under the constraints: } \\
R_{n}\left(\sum_{e=1}^{E^{H}} G_{e n}^{H} Q_{e}^{t} \beta_{e}\right)=\xi_{n}, \forall n \in F
\end{array}\right.
$$

3.2. Solution of Problem 3.11. The constrained problem (3.11) can be solved by a Lagrange multiplier method. Let us introduce column vectors $\xi$ and $\rho$ of $\mathbb{R}^{M}$ and matrices $D$ and $T$.

The components of the vector $\rho$ are Lagrange multipliers relative to the constraints in (3.11) and $\xi$ is the vector defined by 3.10 . If we suppose that $F=\left\{i_{1}, \ldots, i_{|F|}\right\}$, $\xi$ and $\rho$ are defined block-wise as:

$$
\xi=\left(\begin{array}{c}
\xi_{i_{1}} \\
\vdots \\
\xi_{i_{|F|}}
\end{array}\right), \quad \rho=\left(\begin{array}{c}
\rho_{i_{1}} \\
\vdots \\
\rho_{i_{|F|}}
\end{array}\right) \text { with } \xi_{i_{n}}, \rho_{i_{n}} \in \mathbb{R}^{\left|J_{n}\right|}
$$

The matrix $D$ of dimension $(\widetilde{M}, \widetilde{M})$ is the block-diagonal matrix whose diagonal blocks are the matrices $K_{e}, e \in\left\{1, \ldots, E^{H}\right\}$. Finally, the matrix $T$ maps a vector from $\mathbb{R}^{M}$ to $\mathbb{R}^{\widetilde{M}}$ in the following way:

$$
T: \rho \mapsto\left(\begin{array}{c}
Q_{1}\left(\sum_{n \in F} G_{1 n}^{H} R_{n}^{t} \rho_{n}\right) \\
\vdots \\
Q_{E^{H}}\left(\sum_{n \in F} G_{E^{H} n}^{H} R_{n}^{t} \rho_{n}\right)
\end{array}\right)
$$

The minimization problem 3.11) can now be written:

To find $\bar{\beta}_{c} \in \mathbb{R}^{\widetilde{M}}$ minimizing $\bar{\beta}^{t} D \bar{\beta}$ in $\mathbb{R}^{\widetilde{M}}$ under the constraint $T^{t} \bar{\beta}=\xi$, or by introducing Lagrange multipliers:

$$
\left\{\begin{array}{l}
\text { To find a critical point }\left(\bar{\beta}_{c}, \rho_{c}\right) \in \mathbb{R}^{\widetilde{M}} \times \mathbb{R}^{M} \text { of the Lagrangian } \mathcal{L} \text { defined by: } \\
\mathcal{L}(\bar{q} \beta, \rho)=\frac{1}{2} \bar{\beta}^{t} D \bar{\beta}+\rho^{t}\left(\xi-T^{t} \bar{\beta}\right) .
\end{array}\right.
$$

This critical point must satisfy the system of equations:

$$
\left(\begin{array}{cc}
D & -T \\
T^{t} & 0
\end{array}\right)\left(\begin{array}{l}
\bar{\beta} \\
\rho
\end{array}\right)=\left(\begin{array}{l}
0 \\
\xi
\end{array}\right)
$$

This linear system can be solved in the following way: 
- first, the vector $\rho_{c}$ of Lagrange multipliers is determined by applying an iterative method to the system:

$$
T^{t} D^{-1} T \rho=\xi
$$

As we will see in Subsection 4.2, the matrix $T^{t} D^{-1} T$ in (3.17) is symmetric positive definite; the construction of the $R_{n}$ 's will precisely ensure this property.

- then, we return to the computation of $\bar{\beta}_{c}$ by solving:

$$
D \bar{\beta}=T \rho_{c} .
$$

The matrix $T^{t} D^{-1} T$ will not be assembled and its multiplication by a vector can be made efficiently; details will be given in Subsection 5.1.

\section{Elements required by the construction.}

4.1. Algebraic decomposition into subdomains. Associated to the fine nodal basis $\left(\phi_{p}^{h}\right)_{p=1, \ldots, N^{h}}$, we give an oriented simple connected graph by its set of vertices indexed by $\left\{1, \ldots, N^{h}\right\}$ and its set of edges $\mathcal{S}^{h} \subset\left\{1, \ldots, N^{h}\right\}^{2}$. The orientations of the edges are arbitrary; there are no loops i.e. no elements $(p, p)$ in $\mathcal{S}^{h}$ and if $(p, q)$ belongs to $\mathcal{S}^{h},(q, p)$ does not belong to $\mathcal{S}^{h}$. By convention, $p$ is the origin and $q$ is the end of the edge $(p, q)$. The edges could be the geometric edges of the mesh, but we do not restrict ourselves to this situation: starting from the second finest grid, we will have no more geometric edges.

The edges are numbered by $i \in\left\{1, \ldots, E^{h}\right\}$, where $E^{h}=\left|\mathcal{S}^{h}\right|$; the $i$-th edge is denoted by $(p(i), q(i))$ and conversely if $p=p(i)$ and $q=q(i)$, we will write $i=\overline{p q}^{h}$.

Let us now give the precise definition of the edge-node incidence matrix $G^{h}$ of size $E^{h} \times N^{h}$ :

$$
G_{i l}^{h}=\left\{\begin{aligned}
-1 & \text { if } l=p(i) \\
+1 & \text { if } l=q(i) \\
0 & \text { otherwise. }
\end{aligned}\right.
$$

Two assumptions on the fine bases are supposed to be satisfied at the beginning. We suppose that the fine nodal basis $\left(\phi_{p}^{h}\right)_{p=1, \ldots, N^{h}}$ satisfies the property:

$$
\sum_{p=1}^{N^{h}} \phi_{p}^{h}(x)=1, \forall x \in \bar{\Omega} .
$$

We assume that there exists a set of subdomains $\left(\Omega_{p}^{h}\right)_{p=1, \ldots, N^{h}}$ of $\Omega$ such that:

$$
\Omega=\bigcup_{p=1}^{N^{h}} \Omega_{p}^{h} \text { and }\left(\bigcup_{q \neq p} \Omega_{q}^{h}\right)^{c} \neq \emptyset, \forall p \in\left\{1, \ldots, N^{h}\right\},
$$

where the superscript $c$ is the standard set-complement, and satisfying :

$$
\begin{aligned}
\operatorname{supp}\left(\phi_{p}^{h}\right) & \subset \overline{\Omega_{p}^{h}}, \forall p \in\left\{1, \ldots, N^{h}\right\} \\
\text { and } \operatorname{supp}\left(\lambda_{i}^{h}\right) & \subset \overline{\Omega_{p}^{h} \cap \Omega_{q}^{h}} \text { if } i=\overline{p q}^{h} .
\end{aligned}
$$

Assumptions (4.3) and (4.4) on the subdomains $\left(\Omega_{p}^{h}\right)_{p=1, \ldots, N^{h}}$ enable us to control the supports of the nodal (edge) functions and to avoid the linear dependence of these functions. 
4.1.1. Notations and principle of the construction for the coarse nodal basis. Several steps have to be considered:

1. In order to localize the supports of the coarse nodal functions $\left(\phi_{n}^{H}\right)_{n=1, \ldots, N^{H}}$, we introduce sets $\left(L_{n}\right)_{n=1, \ldots, N^{H}}$ of indices in $\left\{1, \ldots, N^{h}\right\}$ such that:

$$
\bigcup_{n=1}^{N^{H}} L_{n}=\left\{1, \ldots, N^{h}\right\}
$$

and we will write:

$$
\Omega_{n}^{H}=\bigcup_{p \in L_{n}} \Omega_{p}^{h}
$$

Then it is equivalent to state $\operatorname{supp}\left(\phi_{n}^{H}\right) \subset \overline{\Omega_{n}^{H}}$, and to require the unknowns $\left(\alpha_{p n}\right)$ in 2.7a) to satisfy:

$$
\alpha_{p n}=0, \forall n \in\left\{1, \ldots, N^{H}\right\}, \forall p \in\left\{1, \ldots, N^{h}\right\} \backslash L_{n} .
$$

We introduce a reciprocal set-valued function $\widetilde{L}$ defined by:

$$
\widetilde{L}_{p}=\left\{n \in\left\{1, \ldots, N^{H}\right\} \mid p \in L_{n}\right\}, \forall p \in\left\{1, \ldots, N^{h}\right\} .
$$

Because of (4.5), $\widetilde{L}_{p}$ is non empty. Observe that (4.7) can be rewritten:

$$
\alpha_{p n}=0, \forall p \in\left\{1, \ldots, N^{h}\right\}, \forall n \in\left\{1, \ldots, N^{H}\right\} \backslash \widetilde{L}_{p} .
$$

An illustration of these definitions is given in Figure 4.1. For the fine graph in Figure 1.1(a), we set $L_{1}=\{1,2,3,4,5,6,7\}, L_{2}=\{5,6,8,9,13,14\}$ and $L_{3}=\{7,8,10,11,12\}$. One obtains, for instance, the sets $\widetilde{L}_{7}=\{1,3\}$ and $\widetilde{L}_{4}=\{1\}$.

2. The constant preservation constraint of (3.1) is obtained by enforcing:

$$
\sum_{n=1}^{N^{H}} \alpha_{p n}=1, \forall p \in\left\{1, \ldots, N^{h}\right\} .
$$

Indeed, we substitute $2.7 \mathrm{a}$ ) into the constant preservation constraint and we use (4.2):

$$
\begin{aligned}
\sum_{n=1}^{N^{H}} \phi_{n}^{H}(x) & =\sum_{n=1}^{N^{H}}\left(\sum_{p=1}^{N^{h}} \alpha_{p n} \phi_{p}^{h}(x)\right) \\
& =\sum_{p=1}^{N^{h}} \phi_{p}^{h}(x)\left(\sum_{n=1}^{N^{H}} \alpha_{p n}\right)=\sum_{p=1}^{N^{h}} \phi_{p}^{h}(x)=1 .
\end{aligned}
$$

3. We compute the prolongation matrix $\alpha$ with the encoded support constraint (4.7) by minimizing the energy functional from (3.1) under the constant preservation constraint (4.10). A method is given in 16]. 


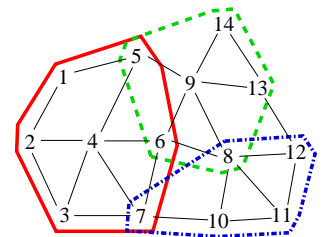

(a) Fine graph with node indices. Sets

$\left(L_{n}\right)_{n=1, \ldots, 3}$ are surrounded.

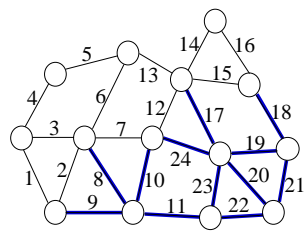

(b) Fine graph with edge indices. Set $C_{3}$ is represented by bold edges.

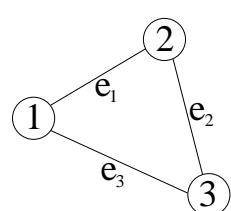

(c) Coarse graph.

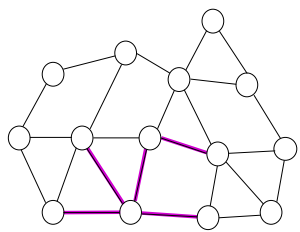

(d) Set $I_{e_{3}}$ is represented by bold edges.

FIG. 4.1. Representation of the fine and coarse graphs, sets $\left(L_{n}\right)_{n=1, \ldots, 3}, C_{3}$ and $I_{e_{3}}$.

4.1.2. More notations and definitions: how to relate coarse and fine, edge and nodal elements. For all $n$ in $\left\{1, \ldots, N^{H}\right\}$, we let $C_{n}$ be a set of fine edge indices; these fine edges start or end in a node whose index is in $L_{n}$ :

$$
C_{n}=\left\{i \in\left\{1, \ldots, E^{h}\right\} \mid i=\overline{p q}^{h} \text { with } p \text { or } q \in L_{n}\right\} .
$$

By (4.4) and (4.6), for $i$ in $C_{n}$, the support of the fine edge basis function $\lambda_{i}^{h}$ is included in the $n$-th coarse nodal domain:

$$
i \in C_{n} \Longrightarrow \operatorname{supp}\left(\lambda_{i}^{h}\right) \in \overline{\Omega_{n}^{H}}
$$

We say that the fine edge function $\lambda_{i}^{h}$ contributes to the gradient of the coarse nodal function $\phi_{n}^{H}$ if $i$ belongs to $C_{n}$. Indeed, from (2.5) and (2.7a), we get:

$$
\operatorname{grad} \phi_{n}^{H}=\sum_{p=1}^{N^{h}} \alpha_{p n}\left(\sum_{i=1}^{E^{h}} G_{i p}^{h} \lambda_{i}^{h}\right)=\sum_{i=1}^{E^{h}}\left(G^{h} \alpha_{\bullet}\right)_{i} \lambda_{i}^{h},
$$

and from (4.1), 4.7) and (4.11):

$$
\left(G^{h} \alpha_{\bullet}\right)_{i}=0, \forall n \in\left\{1, \ldots, N^{H}\right\}, \forall i \in\left\{1, \ldots, E^{h}\right\} \backslash C_{n} .
$$

We introduce the reciprocal set-valued function $\widetilde{C}$ :

$$
\widetilde{C}_{i}=\left\{n \in\left\{1, \ldots, N^{H}\right\} \mid i \in C_{n}\right\}, \forall i \in\left\{1, \ldots, E_{h}\right\} .
$$

In Figure 4.1(b), the fine edges are numbered, set $C_{3}$ is highlighted and we can draw out, for instance, the set $\widetilde{C}_{8}=\{1,3\}$.

According to 4.8, (4.11) and 4.14) we infer:

$$
\widetilde{C}_{i}=\widetilde{L}_{p} \cup \widetilde{L}_{q} \text {, if } i=\overline{p q}^{h} .
$$

As a consequence, $\widetilde{C}_{i}$ cannot be empty and the constant preservation constraint 4.10 ) with (4.9) becomes:

$$
\sum_{n \in \widetilde{C}_{i}} \alpha_{p n}=\sum_{n \in \widetilde{C}_{i}} \alpha_{q n}=1 \text {, if } i=\overline{p q}^{h} .
$$

By analogy with the beginning of Subsection 4.1, we let $\mathcal{S}^{H}$ be the set of edges of a simple oriented connected graph whose vertices are indexed by $\left\{1, \ldots, N^{H}\right\}$; the 
elements of $\mathcal{S}^{H}$ are indexed by $e \in\left\{1, \ldots, E^{H}\right\}$, and the inverse of the bijection $e \mapsto(n(e), m(e))$ is $(n, m) \mapsto \overline{n m}^{H}$. Similarly to the matrix $G^{h}$, we introduce the coarse edge-node incidence matrix $G^{H}$ of dimension $E^{H} \times N^{H}$ with $E^{H}=\left|\mathcal{S}^{H}\right|$.

The set $\mathcal{S}^{H}$ has to satisfy a compatibility relation with the sets $C_{n}$ :

$$
C_{n} \cap C_{m} \neq \emptyset, \forall(n, m) \in \mathcal{S}^{H} .
$$

For $e$ in $\left\{1, \ldots, E^{H}\right\}$, we define the non empty index set $I_{e}$ of the coarse edge corresponding to $e=\overline{n m}^{H}$ :

$$
I_{e}=C_{n} \cap C_{m} \text { if } e=\overline{n m}^{H} .
$$

Then from (4.12), if $i$ belongs to $I_{e}$, the fine edge function $\lambda_{i}^{h}$ has its support included in $\overline{\mathcal{U}_{e}}$ :

$$
i \in I_{e} \Longrightarrow \operatorname{supp}\left(\lambda_{i}^{h}\right) \subset \overline{\mathcal{U}_{e}} \text { with } \mathcal{U}_{e}=\Omega_{n}^{H} \cap \Omega_{m}^{H} .
$$

The coarse graph in Figure 4.1(c) is related to the fine graph in Figure 4.1(a). Set $I_{e_{3}}$ is represented in Figure $4.1(\mathrm{~d})$.

In order to satisfy the support constraint $\operatorname{supp}\left(\lambda_{e}^{H}\right) \subset \overline{\mathcal{U}_{e}}$, we impose on the unknowns $\left(\beta_{i e}\right)$ in $(2.7 \mathrm{~b})$ the constraints:

$$
\beta_{i e}=0, \forall e \in\left\{1, \ldots, E^{H}\right\}, \forall i \in\left\{1, \ldots, E^{h}\right\} \backslash I_{e} .
$$

By introducing the reciprocal set-valued function $\widetilde{I}$ defined by:

$$
\widetilde{I}_{i}=\left\{e \in\left\{1, \ldots, E^{H}\right\} \mid i \in I_{e}\right\}, \forall i \in\left\{1, \ldots, E^{h}\right\},
$$

the relation $(4.19)$ can be rewritten as:

$$
\beta_{i e}=0, \forall i \in\left\{1, \ldots, E^{h}\right\}, \forall e \in\left\{1, \ldots, E^{H}\right\} \backslash \widetilde{I}_{i} .
$$

The set $\widetilde{I}_{i}$ might be empty for some $i \in\left\{1, \ldots, E^{h}\right\}$ i.e. some fine edge function might not contribute to any coarse edge function but, as the sets $I_{e}$ are never empty, we get:

$$
\bigcup_{i=1}^{E^{h}} \widetilde{I}_{i}=\left\{1, \ldots, E^{H}\right\} .
$$

4.1.3. Graph- and set-theoretical properties of $\widetilde{I}$ and $\widetilde{C}$. We will show that it is not necessary to enforce the constraints $(2.8 \mathrm{~b})$ for all $(i, n)$. We need the following lemma which is a direct consequence of (4.14), (4.18) and (4.20):

LEMMA 4.1. If $\widetilde{I}_{i}$ is non empty, the following equivalence holds:

$$
\overline{m n}^{H} \in \widetilde{I}_{i} \Leftrightarrow(m, n) \in \mathcal{S}^{H} \text { and }\{m, n\} \subset \widetilde{C}_{i} .
$$

Thus the edges indexed by $\widetilde{I}_{i}$ are the edges of $\mathcal{S}^{H}$ whose extremity nodes are indexed by $\widetilde{C}_{i}$. From this result, we will first deduce that the relations in $(2.8 \mathrm{~b})$ are always fulfilled in the case $n \notin \widetilde{C}_{i}$.

Proposition 4.2. For every $i$ in $\left\{1, \ldots, E^{h}\right\}$ and every $n$ in $\left\{1, \ldots, N^{H}\right\} \backslash \widetilde{C}_{i}$, the constraints (2.8b) of index $(i, n)$ are implicitly satisfied. 
Proof. According to (4.13), the $(i, n)$ coefficient of the right-hand side of (2.8b) vanishes.

Conversely, according to 4.21 ) the left-hand side of (2.8b) is given by:

$$
\sum_{e \in \widetilde{I}_{i}} \beta_{i e} G_{e n}^{H}
$$

It vanishes if $\widetilde{I}_{i}$ is empty. If $e=\overline{l m}^{H}$ belongs to $\widetilde{I}_{i}$, Lemma 4.1 implies that $l$ and $m$ belong to $\widetilde{C}_{i}$. However for $G_{e n}^{H}$ not to vanish, $m$ and $l$ must be equal to $n$ and this contradicts the assumption $n \notin \widetilde{C}_{i}$.

Secondly, the following proposition points out that some relations in (2.8b) are linearly dependent.

Proposition 4.3. Let $i$ be a fixed index in $\left\{1, \ldots, E^{h}\right\}$ and $m$ some index in $\widetilde{C}_{i}$. If the constraints (2.8b) of index $(i, n)$ are satisfied for all $n$ in $\widetilde{C}_{i} \backslash\{m\}$, the constraint of index $(i, m)$ is also satisfied.

Proof. It is sufficient to prove the equality:

$$
\sum_{n \in \widetilde{C}_{i}}\left(\sum_{e=1}^{E^{H}} \beta_{i e} G_{e n}^{H}\right)=\sum_{n \in \widetilde{C}_{i}}\left(\sum_{r=1}^{N^{h}} G_{i r}^{h} \alpha_{r n}\right) .
$$

For $i=\overline{p q}^{h}$, according to (4.1) and (4.16), the right-hand side vanishes since:

$$
\sum_{n \in \widetilde{C}_{i}}\left(\sum_{r=1}^{N^{h}} G_{i r}^{h} \alpha_{r n}\right)=\sum_{n \in \widetilde{C}_{i}} \alpha_{q n}-\sum_{n \in \widetilde{C}_{i}} \alpha_{p n} .
$$

For the left-hand side, according to 4.21), it comes:

$$
\sum_{n \in \widetilde{C}_{i}}\left(\sum_{e=1}^{E^{H}} \beta_{i e} G_{e n}^{H}\right)=\sum_{e \in \widetilde{I}_{i}} \beta_{i e}\left(\sum_{n \in \widetilde{C}_{i}} G_{e n}^{H}\right) .
$$

It vanishes if $\widetilde{I}_{i}=\emptyset$ and also if $\widetilde{I}_{i} \neq \emptyset$ since for all $e$ in $\widetilde{I}_{i}$, by Lemma 4.1 and the definition of $G^{H}$ :

$$
\sum_{n \in \widetilde{C}_{i}} G_{e n}^{H}=\sum_{n=1}^{N^{H}} G_{e n}^{H}=0 .
$$

$\square$

4.2. How to choose the $R_{n}$ 's in order to simplify the computational process and to have a unique coarse edge basis. The choice of the index set $J_{n}$ and therefore the projections $R_{n}$ can now be explicitly defined.

All the matrices $\left(K_{e}\right)_{e=1, \ldots, E^{H}}$ are assumed to be symmetric positive definite; from the definition of the matrix $D$ (Subsection 3.2), it is clear that $D^{-1}$ is symmetric positive definite. Therefore, in order to obtain a unique solution of (3.17), the matrix $T$ must have full column rank, which has to be enforced by a proper choice of the $R_{n}$ 's. If $T$ does not satisfy this property, the set of $\beta$ satisfying (2.9) can be empty and the existence of a solution to (3.17) is not ensured. 
We define the reciprocal set-valued function $\widetilde{J}$ :

$$
\widetilde{J}_{i}=\left\{n \in\left\{1, \ldots, N^{H}\right\} \mid i \in J_{n}\right\}, \forall i \in\left\{1, \ldots, E^{h}\right\} .
$$

Since the sets $\widetilde{I}_{i}$ and $\widetilde{J}_{i}$ may be empty, we introduce the set $\widetilde{F}$ :

$$
\widetilde{F}=\left\{i \in\left\{1, \ldots, E^{h}\right\} \mid \widetilde{I}_{i} \neq \emptyset \text { and } \widetilde{J}_{i} \neq \emptyset\right\} .
$$

Then, for $i$ in $\widetilde{F}$, we can define $\widetilde{G}^{H, i}$ the matrix of dimensions $\left|\widetilde{I}_{i}\right| \times\left|\widetilde{J}_{i}\right|$ extracted from the matrix $G^{H}$ by keeping only the rows $e \in \widetilde{I}_{i}$ and the columns $n \in \widetilde{J}_{i}$.

Proposition 4.4. The following conditions are necessary and sufficient for $T$ to be one-to-one:

$$
\begin{aligned}
& \operatorname{ker}\left(\widetilde{G}^{H, i}\right)=\{0\}, \forall i \in \widetilde{F} \\
& \forall i \in\left\{1, \ldots, E^{h}\right\}, \widetilde{I}_{i}=\emptyset \Rightarrow \widetilde{J}_{i}=\emptyset
\end{aligned}
$$

Proof. Let $\rho$ be in $\mathbb{R}^{M}$. For $n$ in $F$ defined in (3.7), we observe that:

$$
R_{n}^{t} \rho_{n}=\sum_{i \in J_{n}} \rho_{n, i} u_{i}
$$

where $u_{i}$ is the $i$-th vector of the canonical basis of $\mathbb{R}^{E^{h}}$. We let $T$ operate on $\rho$, and for that purpose we look at the block-wise result. Let $e$ be in $\left\{1, \ldots, E^{H}\right\}$, by the definition of $T$ in $(3.13)$ :

$$
\begin{aligned}
(T \rho)_{e} & =Q_{e}\left(\sum_{n \in F} G_{e n}^{H}\left(\sum_{i \in J_{n}} \rho_{n, i} u_{i}\right)\right), \\
& =\sum_{n=1}^{N^{H}} \sum_{i \in J_{n}} G_{e n}^{H} \rho_{n, i} Q_{e}\left(u_{i}\right) .
\end{aligned}
$$

From (4.22), the components of $\rho$ indexed by the set of couples $(n, i)$ with $n$ in $\left\{1, \ldots, N^{H}\right\}$ and $i$ in $J_{n}$ can equivalently be indexed by $i$ in $\left\{1, \ldots, E^{h}\right\}$ and $n$ in $\widetilde{J}_{i}$. Thus $(T \rho)_{e}$ can be rewritten as:

$$
(T \rho)_{e}=\sum_{i=1}^{E^{h}}\left(\sum_{n \in \widetilde{J}_{i}} G_{e n}^{H} \rho_{n, i}\right) Q_{e}\left(u_{i}\right)
$$

but $Q_{e}\left(u_{i}\right)$ is equal to 0 if $i$ is not in $I_{e}$, then:

$$
(T \rho)_{e}=\sum_{i \in I_{e}}\left(\sum_{n \in \widetilde{J}_{i}} G_{e, n}^{H} \rho_{n, i}\right) Q_{e}\left(u_{i}\right)
$$

Since the vectors $\left(Q_{e}\left(u_{i}\right)\right)_{i \in I_{e}}$ are linearly independent in $\mathbb{R}^{\left|I_{e}\right|}$, we infer from relation (4.26d):

$$
T \rho=0 \Leftrightarrow \sum_{n \in \widetilde{J}_{i}} G_{e, n}^{H} \rho_{n, i}=0, \forall e \in\left\{1, \ldots, E^{H}\right\}, \forall i \in I_{e}
$$


Remarking that $i \in I_{e}$ corresponds to $e \in \widetilde{I}_{i}$, 4.27a becomes:

$$
T \rho=0 \Leftrightarrow \sum_{n \in \widetilde{J}_{i}} G_{e, n}^{H} \rho_{n, i}=0, \forall i \in \widetilde{F}, \forall e \in \widetilde{I}_{i}
$$

which can be written, according to the definition of $\widetilde{G}^{H, i}$, as:

$$
T \rho=0 \Leftrightarrow \widetilde{G}^{H, i} \rho^{i}=0, \forall i \in \widetilde{F},
$$

where $\rho^{i} \in \mathbb{R}^{\left|\widetilde{J}_{i}\right|}$ is of components $\rho_{n, i}$ for $n$ in $\widetilde{J}_{i}$. Finally, one obtains:

$$
T \rho=0 \Leftrightarrow \rho^{i} \in \operatorname{ker}\left(\widetilde{G}^{H, i}\right), \forall i \in \widetilde{F} \text {. }
$$

We can now prove the sufficiency of (4.24). If we assume condition $(4.24 \mathrm{~b})$ is satisfied then:

$$
\widetilde{F}=\left\{i \in\left\{1, \ldots, E^{h}\right\} \mid \widetilde{J}_{i} \neq \emptyset\right\},
$$

and $\rho$, vector of $\mathbb{R}^{M}$, is defined by the knowledge of its blocks $\rho^{i}$ for all $i$ in $\widetilde{F}$. Condition (4.24a) associated with relation (4.28) enables us to conclude:

$$
T \rho=0 \Rightarrow \rho=0
$$

Then, conditions (4.24) are sufficient for $T$ to be one-to-one.

Conversely, assume that:

$$
\exists i_{0} \in \widetilde{F}, \operatorname{ker}\left(\widetilde{G}_{i_{0}}^{H}\right) \neq\{0\},
$$

Let $\rho$ be in $\mathbb{R}^{M} \backslash\{0\}$ such that:

$$
\rho^{j}=\{0\}, \forall j \neq i_{0} \text { and } \rho^{i_{0}} \in \operatorname{ker}\left(\widetilde{G}_{i_{0}}^{H}\right) \backslash\{0\} .
$$

By relation (4.28), $\rho$ is in $\operatorname{ker}(T)$. Hence, condition (4.24a $)$ is necessary for $T$ to be one-to-one.

In the same way, assume that:

$$
\exists i_{0} \in\left\{1, \ldots, E^{h}\right\}, \widetilde{I}_{i_{0}}=\emptyset \text { and } \widetilde{J}_{i_{0}} \neq \emptyset,
$$

Let $\rho$ in $\mathbb{R}^{M} \backslash\{0\}$ be such that:

$$
\rho^{j}=\{0\}, \forall j \neq i_{0} .
$$

Since $i_{0}$ is not in $\widetilde{F}, \rho$ is in $\operatorname{ker}(T)$ from 4.28 . Condition $4.24 \mathrm{~b}$ is necessary for $T$ to be one-to-one. $\square$

In order to reduce the number of enforced constraints, from Propositions 4.2 and 4.3, a suitable choice of sets $\widetilde{J}_{i}$ is

$$
\widetilde{J}_{i}=\widetilde{C}_{i} \backslash\{m\}, \text { with } m \in \widetilde{C}_{i}, \forall i \in\left\{1, \ldots, E^{h}\right\} .
$$

For such a choice, the condition of Proposition 4.4 can be given in terms of connectivity of induced subgraphs of the graph of edges in $\mathcal{S}^{H}$. More precisely, for 
any fine edge $i$ of $\mathcal{S}^{h}$, let $\mathcal{S}^{H, i}$ be the induced subgraph of the graph of edges in $\mathcal{S}^{H}$, whose vertices are indexed by $\widetilde{C}_{i}$ and edges by $\widetilde{I}_{i}$.

Corollary 4.5. For $\widetilde{J}_{i}=\widetilde{C}_{i} \backslash\{m\}, i=1, \ldots, E^{h}, T$ is one-to-one iff for all $i$ in $\left\{1, \ldots, E^{h}\right\}$ the induced subgraph $\mathcal{S}^{H, i}$ is connected.

Proof. Observe first that $\widetilde{J}_{i}$ is empty if and only if the subgraph $\mathcal{S}^{H, i}$ reduces to one vertex which happens by Lemma 4.1 if $\widetilde{I}_{i}$ is empty.

Assume now $\widetilde{I}_{i}$ is non empty. By Lemma 4.1 the edge-node incidence matrix of the subgraph is deduced from $G^{H}$ by keeping only the rows $e \in \widetilde{I}_{i}$ and the columns $n \in \widetilde{C}_{i}$. By deleting one column of index $m \in \widetilde{C}_{i}$, the obtained matrix $\widetilde{G}^{H, i}$ satisfies the condition (4.24a of Proposition 4.4 iff the subgraph $\mathcal{S}^{H, i}$ is connected.

The following subsection is devoted to the construction of index sets $L_{n}$ and an incidence matrix $G^{H}$ which satisfy the connectivity condition on the induced subgraph $\mathcal{S}^{H, i}$.

4.3. Construction of the index sets $L_{n}$. We start from an $N^{h} \times N^{h}$ nodal symmetric matrix $B^{h}$. When passing from a fine level to a coarser level, we just require:

$$
B_{p p}^{h} \neq 0, \forall p \in\left\{1, \ldots, N^{h}\right\}
$$

and a compatibility condition with the set $\mathcal{S}^{h}$ :

$$
(p, q) \in \mathcal{S}^{h} \Longrightarrow B_{p q}^{h} \neq 0 .
$$

This condition means that $B_{p q}^{h}$ must not vanish on the couples $(p, q)$ corresponding to the edges belonging to $\mathcal{S}^{h}$, but it might also be different from zero on other couples. In other words, there may be more edges $(p, q)$ determined by the condition $B_{p q}^{h} \neq 0$ than elements $(p, q)$ or $(q, p)$ in $\mathcal{S}^{h}$.

As Reitzinger and Schöberl do in [14, we define a map:

$$
\text { ind } \begin{aligned}
\mathbb{R}^{N^{h}} & \rightarrow \mathbb{R}^{N^{H}} \\
p & \mapsto \operatorname{ind}(p) .
\end{aligned}
$$

The inverse images of $n$ by the mapping ind make up a partition of $\left\{1, \ldots, N^{h}\right\}$ into sets. The set $H_{n}$ is the aggregate of fine indices indexed by $n$ :

$$
H_{n}=\left\{p \in\left\{1, \ldots, N^{h}\right\} \mid \operatorname{ind}(p)=n\right\},
$$

An example of a fine graph with the partition of the nodes is given in Figure 4.2(a). The $H_{n}$ 's make up an arbitrary partition of $\left\{1, \ldots, N^{h}\right\}$, but there are algorithms for choosing good partitions, for instance the aggregation algorithm proposed in 15 , Section 5].

For every fine index $p$ in $\left\{1, \ldots, N^{h}\right\}$, we define:

$$
\mathcal{N}_{p}=\left\{q \in\left\{1, \ldots, N^{h}\right\} \mid B_{p q}^{h} \neq 0\right\}
$$

This is the set of all the $B^{h}$-neighbors of the nodal variable $p$ and the variable itself. From the compatibility relation (4.30), we remark that:

$$
(p, q) \in \mathcal{S}^{h} \Longrightarrow q \in \mathcal{N}_{p} \text {. }
$$


For all $n$ in $\left\{1, \ldots, N^{H}\right\}$, we will write:

$$
L_{n}=\bigcup_{p \in H_{n}} \mathcal{N}_{p}
$$

Observe that the set $\widetilde{L}_{p}$ defined in 4.8 is related to $\mathcal{N}_{p}$ as follows:

$$
\widetilde{L}_{p}=\operatorname{ind}\left(\mathcal{N}_{p}\right) \text {. }
$$

We also infer from (4.33) and the symmetry of $B^{h}$ :

$$
p \in \mathcal{N}_{q} \Leftrightarrow q \in \mathcal{N}_{p}
$$

Some sets $L_{n}$ corresponding to the partition of Figure 4.2(a) are represented in Figure $4.2(\mathrm{~b})$.

REMARK 4.6. On the initial mesh, some choices for B are proposed by Reitizinger and Kaltenbacher in 7 . Observe that the simplest way to take into account the connectivity of the initial mesh and to satisfy conditions (4.29) and (4.30) is to put $B^{h}=\left(G^{h}\right)^{t} G^{h}$. For the coarser level, the matrix can be constructed from the Galerkin product $\alpha^{t} B^{h} \alpha$.

4.4. Definition of a compatible coarse edge incidence matrix $G^{H}$. By analogy with Reitzinger and Schöberl in [14], we choose $\mathcal{S}^{H}$ such that $(n, m)$ or $(m, n)$ is an edge if there exists $p \in H_{n}$ and $q \in H_{m}$ defined in (4.32) such that $p$ and $q$ are $B^{h}$-neighbors, i.e.:

$$
\begin{array}{r}
\mathcal{S}^{H}=\left\{(n, m) \in\left\{1, \ldots, N^{H}\right\}^{2} \mid n \neq m, \exists p, q \in\left\{1, \ldots, N^{h}\right\}\right. \\
\text { with } \left.\operatorname{ind}(p)=n, \operatorname{ind}(q)=m, p<q \text { and } B_{p q}^{h} \neq 0\right\} .
\end{array}
$$

From this definition, we easily verify that the connectivity property of the fine graph $\mathcal{S}^{h}$ remains for the coarse graph $\mathcal{S}^{H}$. Observe also that $B_{p q}^{h} \neq 0$ implies that $p$ is in $L_{\text {ind }(p)} \cap L_{\operatorname{ind}(q)}$. Then $C_{\text {ind }(p)} \cap C_{\text {ind }(q)}$ is non empty and $\mathcal{S}^{H}$ satisfies condition (4.17).

The coarse graph coming from the graph partition of Figure 4.2(a) is represented in Figure 4.2(c). Some sets $\widetilde{L}_{p}$ are also represented in Figure $4.2(\mathrm{~d})$.

For proving the connectivity of the induced graph $\mathcal{S}^{H, i}$, we need an intermediate result.

LEMMA 4.7. Let $p \in\left\{1, \ldots, N^{h}\right\}$ be a fine nodal index and assume that the coarse nodal set $\widetilde{L}_{p}$ has at least two elements. Then $\operatorname{ind}(p)$ is connected to the other vertices in $\widetilde{L}_{p}$ by an edge of $\mathcal{S}^{H}$.

Proof. Let $n$ be in $\widetilde{L}_{p} \backslash\{\operatorname{ind}(p)\}$. By 4.36, there exists $p_{n}$ in $\mathcal{N}_{p}$ such that $n=\operatorname{ind}\left(p_{n}\right)$, then:

$$
B_{p p_{n}}^{h} \neq 0 \text { and } \operatorname{ind}(p) \neq \operatorname{ind}\left(p_{n}\right)
$$

From definition 4.37) of $S^{H}$, we conclude that $(n, \operatorname{ind}(p))$ or $(\operatorname{ind}(p), n)$ is in $\mathcal{S}^{H}$.

Proposition 4.8. For all $i$ in $\left\{1, \ldots, E^{h}\right\}$, the coarse graph $\mathcal{S}^{H, i}$ is connected.

Proof. Let $i=\overline{p q}^{h}$; then from (4.15) $\widetilde{C}_{i}=\widetilde{L}_{p} \cup \widetilde{L}_{q}$. From (4.34) and (4.36), we infer:

$$
\{\operatorname{ind}(p), \operatorname{ind}(q)\} \subset \widetilde{L}_{p} \cap \widetilde{L}_{q}
$$




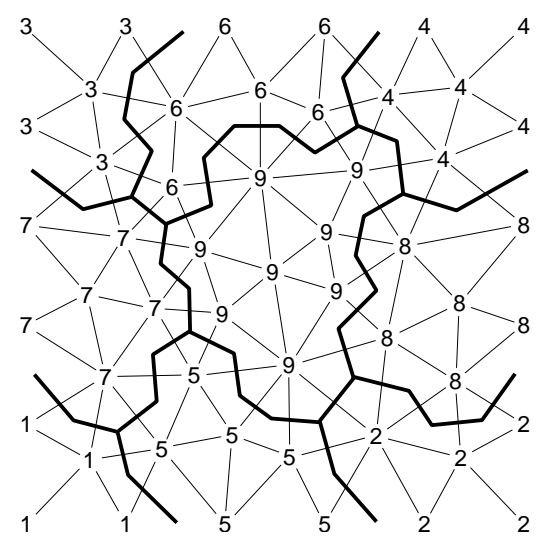

(a) Initial graph and partition

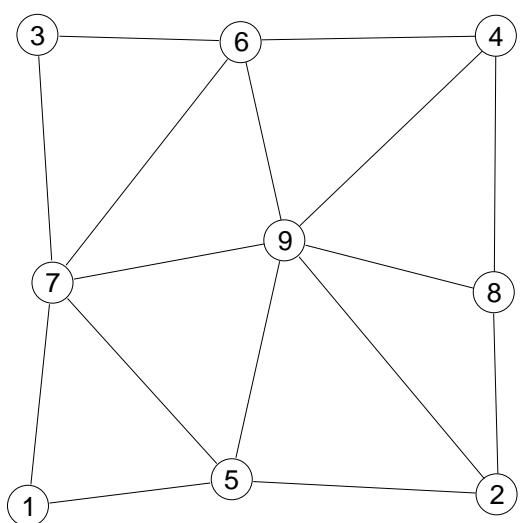

(c) Coarse graph for partition $4.2(\mathrm{a})$

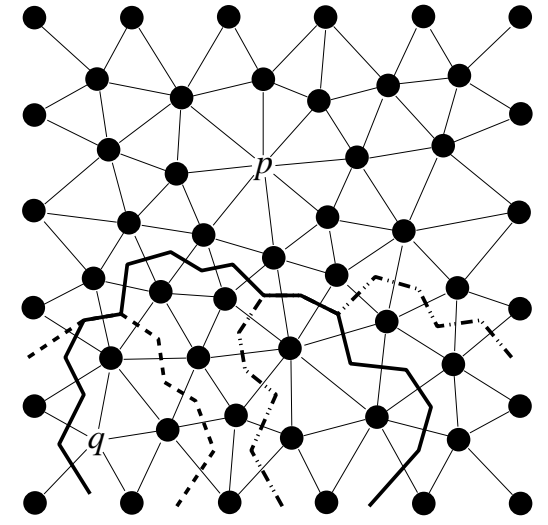

(b) Representation of $L_{1}, L_{5}$ and $L_{2}$.

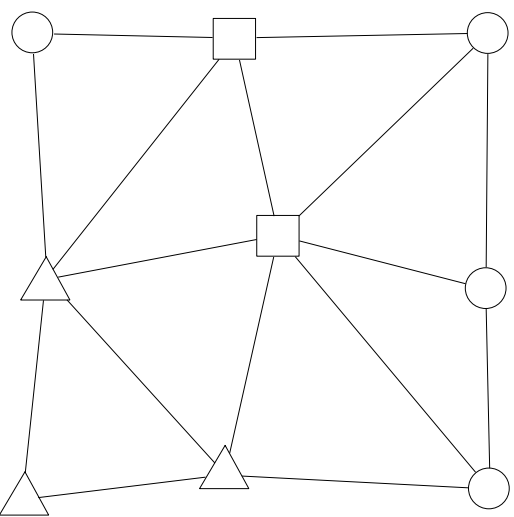

(d) Representation of $\widetilde{L}_{q}(\triangle)$ and $\widetilde{L}_{p}(\square)$. Nodes $p$ and $q$ are in Fig. 4.2(b)

FIG. 4.2. Fig. 4.2(a): Initial graph and partition of nodes in sets $H_{n}$, Fig. 4.2(b): some sets $L_{n}$, Fig. 4.2(c): construction of the coarse graph corresponding to the partition in Fig. 4.2(c) and Fig. 4.2(d): representation of the sets $\widetilde{L}_{q}$ and $\widetilde{L}_{p}$ referring to Fig. 4.2(b).

Assume first ind $(p) \neq \operatorname{ind}(q)$. Lemma 4.7 implies that ind $(p)$ and ind $(q)$ are connected and for all $n$ in $\widetilde{C}_{i} \backslash\{\operatorname{ind}(r)\}$ with $r \in\{p, q\}, n$ and ind $(r)$ are connected.

Assume now $\operatorname{ind}(p)=\operatorname{ind}(q)=n$ with $\left|\widetilde{C}_{i}\right|>1$. From Lemma 4.7, we infer:

$$
\forall m \in \widetilde{C}_{i} \backslash\{n\}, n \text { and } m \text { are connected. }
$$

$\square$

REMARK 4.9. Reitzinger and Schöberl's approach in [14] can be described with our notations. They chose $L_{n}=H_{n}$ for all $n$ in $\left\{1, \ldots, N^{H}\right\}$ and the coarse graph $G^{H}$ defined in (4.37). This leads to $\left|\widetilde{L}_{p}\right|=1$ for all fine nodal index $p$ and it implies $\left|\widetilde{C}_{i}\right|=1$ or $\left|\widetilde{C}_{i}\right|=2$ with $\left|\widetilde{I}_{i}\right|=1$. Our construction gives larger edge aggregates, which is a nice situation because in the end few fine edges will not contribute to the computation of the coarse edge basis.

REMARK 4.10. For two nested meshes $\tau^{h}$ and $\tau^{H}$, if we take $L_{n}$ as the set of indices of the $\tau^{h}$-neighbors of the coarse node of index $n$ and $G^{H}$ as the matrix natu- 
rally associated with the coarse mesh $\tau^{H}$, the connectivity condition for Corollary 4.5 is clearly satisfied.

\section{Numerical experiments.}

5.1. Matrix multiplication algorithm. In order to solve the linear system (3.17), a non-preconditioned conjugate gradient algorithm is used. Thus only the multiplication of $T^{t} D^{-1} T$ by a vector of $\mathbb{R}^{M}$ is required. We can compute $T^{t} D^{-1} T \rho=$ $\widetilde{\rho}$ in three steps:

- Step 1: For $e=1, \ldots, E^{H}$, compute:

$$
b_{e}=Q_{e}\left(\sum_{n \in F} G_{e n}^{H} R_{n}^{t} \rho_{n}\right)=Q_{e}\left(R_{m}^{t} \rho_{m}-R_{n}^{t} \rho_{n}\right), \text { for } e=\overline{n m}^{H} .
$$

- Step 2: For $e=1, \ldots, E^{H}$, solve:

$$
K_{e} x_{e}=b_{e}
$$

- Step 3: For $n=1, \ldots, N^{H}$, compute:

$$
\tilde{\rho}_{n}=R_{n}\left(\sum_{e=1}^{E^{H}} G_{e n}^{H} Q_{e}^{t} x_{e}\right)
$$

The step 1 requires $\widetilde{M}$ additions and the number of arithmetical operations in step 3 is bounded by $\max _{n}\left(\sum_{e=1}^{E^{H}}\left|G_{e n}^{H}\right|\right) M$. The most expensive part is the solution of all the local problems: $K_{e} x_{e}=b_{e}$, for all $e$ in $\left\{1, \ldots, E^{H}\right\}$.

Observe that such solutions of local problems are also required for the computation of the prolongation matrix $\bar{\beta}_{c}$ from the Lagrange multiplier vector $\rho_{c}$ by using (3.18). For an exact solution of local problems, a factorization of each matrix $K_{e}$ must be done.

5.2. Choice of the bilinear form $b$. In the following tables, different bilinear forms $b$ are used for the minimisation. For convenience, we define a few abbreviations:

- A refers to the bilinear form $a$ of the problem. With this choice, spatial variations of $\gamma$ and $\delta$ together with mesh heterogeneity are completely taken into account for the construction of the finite element basis.

- $A+G^{h} M_{\phi}^{-1}\left(G^{h}\right)^{t}$ refers to the bilinear form defined from this matrix, $M_{\phi}$ being the mass matrix on nodal elements, with mass lumping; hence $M_{\phi}$ is diagonal. This choice enables us to improve the conditioning of local matrices $K_{e}$ as it will be noted for $2 \mathrm{D}$ simulations.

- $S+\operatorname{reg}(\eta)$ refers to the bilinear form $\int_{\Omega} \operatorname{curl} E \cdot \operatorname{curl} E^{\prime}$ added to a local regularisation depending on the parameter $\eta$; more precisely the matrix of the local problem is $S_{e}+\eta \max \left(\operatorname{diag}\left(S_{e}\right)\right)$ Id where the matrix Id is the identity matrix and $S_{e}$ is the local matrix computed from $\int_{\Omega} \operatorname{curl} E \cdot \operatorname{curl} E^{\prime}$. The focus is to improve the conditioning of the local matrices, the essential part of the original bilinear form $a$ being kept.

- Id refers to the use of the identity matrix for the local problems. Then no factorisation is needed but the original problem is no more taken into account in the definition of $b$.

The annotation GSsym means that one symmetric Gauss-Seidel iteration is performed in the local systems instead of an exact solution in order to significantly reduce the complexity. 
5.3. Structured meshes and constant coefficients. In order to validate the proposed choices of bilinear form $b$, we begin the numerical experiments with structured meshes and constant coefficients in the problem. The coefficients $\delta$ and $\gamma$ are set equal to 1 . A sequence of nested meshes $\left(\tau_{k}^{h}\right)_{k=0, \ldots, 4}$ is constructed by a regular refinement starting from a simple triangular or tetrahedral mesh on the unit square and the unit cube (see Fig. 5.3 and 5.2). The source term is given by non-homogeneous boundary conditions. For each level, the edge prolongation matrix $\beta$ is computed by solving system (3.16) with the right-hand side member $\xi$ defined from the standard nodal prolongation operator [3, Chapter 6]. The sets $L_{n}$ together with the edge-node incidence matrices are defined frome the meshes as described in Remark 4.10.

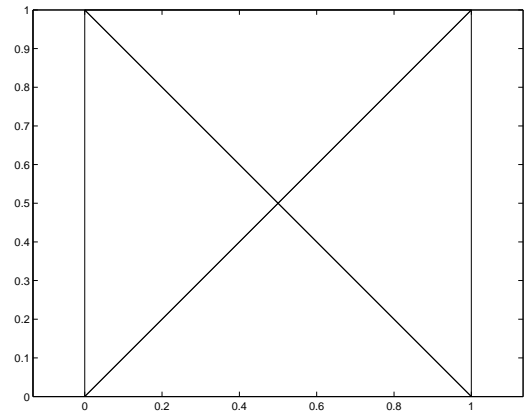

(a)

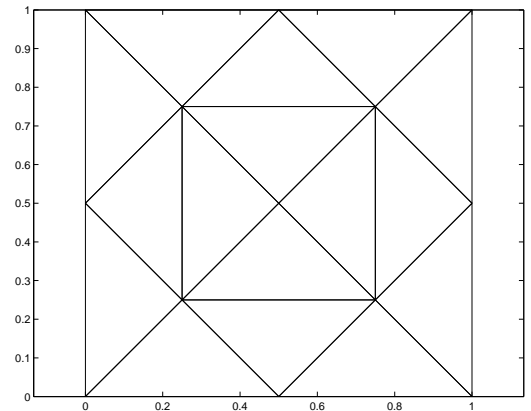

(b)

FIG. 5.1. Initial mesh $\tau_{0}^{h} 5.1(a)$ and first refinement $\tau_{1}^{h} 5.1(b)$.

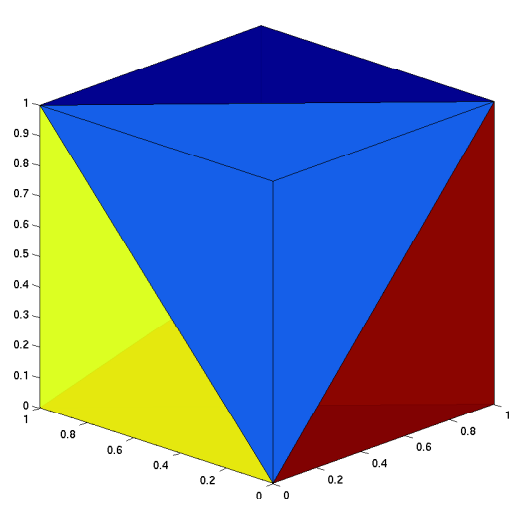

(a)

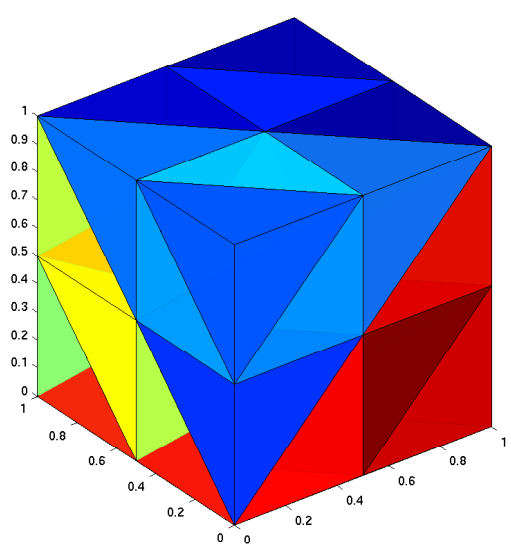

(b)

FIG. 5.2. Initial mesh $\tau_{0}^{h}$ 5.2(a) and first refinement $\tau_{1}^{h}$ 5.2(b)

5.3.1. Dimensions. For the finest level, the number of Lagrange multipliers $M$ given by the vector $\rho$ are reported in Table 5.1 for the 2D case and Table 5.2 for the $3 \mathrm{D}$ case. The number of unknowns $E^{h}$ for each problem is also recalled. Observe that the number of multipliers is less than twice the number of unknowns in the 2D case. The ratio is slightly larger in $3 \mathrm{D}$ except for problems with few unknowns. 


\begin{tabular}{c|c|c|c|c} 
& $\tau_{1}^{h}$ & $\tau_{2}^{h}$ & $\tau_{3}^{h}$ & $\tau_{4}^{h}$ \\
\hline$M=$ Nb of multipliers & 40 & 152 & 592 & 2336 \\
\hline$E^{h}=$ Nb of unknowns & 20 & 88 & 368 & 1504
\end{tabular}

Number (Nb) of Lagrange multipliers and unknowns - 2D, structured meshes.

\begin{tabular}{c|c|c|c|c} 
& $\tau_{1}^{h}$ & $\tau_{2}^{h}$ & $\tau_{3}^{h}$ & $\tau_{4}^{h}$ \\
\hline$M$ & 164 & 1060 & 7544 & 56752 \\
\hline$E^{h}$ & 26 & 316 & 3032 & 26416
\end{tabular}

Number of Lagrange multipliers and unknowns - 3D, structured meshes.

5.3.2. Computation of Lagrange multipliers. The multipliers are initialized to zero. The number of iterations required to divide the Euclidean norm of the residual by $10^{3}$ during the iterative process is reported in Table 5.3 for the $2 \mathrm{D}$ case and Table 5.4 for the 3D case. These numbers are given for several choices of bilinear forms $b$. We also give the number of iterations for each level: finest level $+\ldots+$ coarsest level.

\begin{tabular}{c|c|c|c|c} 
Norm & $\tau_{1}^{h}$ & $\tau_{2}^{h}$ & $\tau_{3}^{h}$ & $\tau_{4}^{h}$ \\
\hline$A$ & 5 & $42+11$ & $64+42+14$ & $79+64+42+14$ \\
\hline$A(\mathrm{GSsym})$ & 11 & $12+11$ & $12+12+11$ & $11+11+12+11$ \\
\hline$A+G^{h} M_{\phi}^{-1}\left(G^{h}\right)^{t}$ & 4 & $9+7$ & $9+8+7$ & $9+8+8+7$ \\
\hline$A+G^{h} M_{\phi}^{-1}\left(G^{h}\right)^{t}(\mathrm{GSsym})$ & 4 & $9+7$ & $9+8+7$ & $9+8+8+7$ \\
\hline$S+\operatorname{reg}(0.1)$ & 6 & $19+10$ & $19+19+9$ & $18+19+19+10$ \\
\hline Id & 2 & $2+2$ & $2+2+2$ & $2+2+2+2$
\end{tabular}

Number of iterations for the multiplier computation (division of the Euclidean norm of the residual by $\left.10^{3}\right)-2 D$, structured meshes.

\begin{tabular}{c|c|c|c|c} 
Norm & $\tau_{1}^{h}$ & $\tau_{2}^{h}$ & $\tau_{3}^{h}$ & $\tau_{4}^{h}$ \\
\hline$A$ & 24 & $32+13$ & $46+16+15$ & $61+20+17+17$ \\
\hline$A(\mathrm{GSsym})$ & 23 & $25+13$ & $32+16+15$ & $39+20+18+17$ \\
\hline$A+G^{h} M_{\phi}^{-1}\left(G^{h}\right)^{t}$ & 41 & $93+21$ & $181+27+17$ & $381+29+21+16$ \\
\hline$A+G^{h} M_{\phi}^{-1}\left(G^{h}\right)^{t}(\mathrm{GSsym})$ & 26 & $42+18$ & $56+21+15$ & $62+22+16+14$ \\
\hline$S+\operatorname{reg}(0.1)$ & 20 & $22+13$ & $29+15+14$ & $36+18+15+15$ \\
\hline Id & 3 & $3+3$ & $3+3+3$ & $3+3+3+3$
\end{tabular}

Number of iterations for the multiplier computation (division of the Euclidean norm of the residual by $10^{3}$ ) - 3D, structured meshes.

In the $2 \mathrm{D}$ case, the behaviour of the algorithm with the original matrix $A$ is not good since the number of iterations increases with the fineness of the initial mesh. The surprising thing is that the number of iterations becomes constant when a GaussSeidel iteration is used instead of an exact solution. For the other cases, the behaviour is relatively homogeneous and the number of iterations is almost constant. In $3 \mathrm{D}$, we 
notice a slow increase in the number of iterations and the behaviour for the original matrix $A$ is not as bad as in the $2 \mathrm{D}$ case. The behaviour for the matrix Id is the best in both cases.

5.3.3. Solution of system (2.4). System (2.4) is solved by a preconditioned conjugate gradient algorithm. The preconditioner is a multilevel method which uses one pre- and one post-smoothing step by Arnold's smoother [1]; on the coarse grid, a direct solver is used. The conjugate gradient method stops when the Euclidean norm of the residual has been divided by $10^{10}$. Table 5.5 gives the results for the $2 \mathrm{D}$ case and Table 5.6 for the 3D case. The mention "geometric" corresponds to the classical geometric multigrid.

\begin{tabular}{c|c|c|c|c} 
Norm & $\tau_{1}^{h}$ & $\tau_{2}^{h}$ & $\tau_{3}^{h}$ & $\tau_{4}^{h}$ \\
\hline$A$ & 5 & 6 & 7 & 7 \\
\hline$A(\mathrm{GSsym})$ & 5 & 6 & 7 & 9 \\
\hline$A+G^{h} M_{\phi}^{-1}\left(G^{h}\right)^{t}$ & 5 & 6 & 7 & 8 \\
\hline$A+G^{h} M_{\phi}^{-1}\left(G^{h}\right)^{t}(\mathrm{GSsym})$ & 5 & 6 & 7 & 8 \\
\hline$S^{2}+\mathrm{reg}(0.1)$ & 5 & 6 & 7 & 7 \\
\hline Id & 5 & 7 & 9 & 11 \\
\hline geometric TABLE 5.5 & 5 & 6 & 7 & 7
\end{tabular}

Number of iterations of the conjugate gradient preconditioned by a multilevel method (division of the Euclidean norm of the residual by $10^{10}$ ) - 2D, structured meshes.

\begin{tabular}{c|c|c|c|c} 
Norm & $\tau_{1}^{h}$ & $\tau_{2}^{h}$ & $\tau_{3}^{h}$ & $\tau_{4}^{h}$ \\
\hline$A$ & 4 & 7 & 10 & 11 \\
\hline$A(\mathrm{GSsym})$ & 4 & 7 & 10 & 11 \\
\hline$A+G^{h} M_{\phi}^{-1}\left(G^{h}\right)^{t}$ & 4 & 7 & 10 & 11 \\
\hline$A+G^{h} M_{\phi}^{-1}\left(G^{h}\right)^{t}(\mathrm{GSsym})$ & 4 & 7 & 10 & 11 \\
\hline$S^{2}+\mathrm{reg}(0.1)$ & 4 & 7 & 10 & 11 \\
\hline Id & 4 & 7 & 10 & 11 \\
\hline geometric TABLE 5.6 & 4 & 7 & 10 & 11
\end{tabular}

Number of iterations of the conjugate gradient preconditioned by a multilevel method (division of the Euclidean norm of the residual by $10^{10}$ ) - 3D, structured meshes.

Once the multipliers are computed, and for all choices of bilinear forms $b$, the efficacy of the geometric and algebraic multigrid methods are comparable.

5.4. Unstructured meshes and varying coefficients. We consider a $2 \mathrm{D}$ problem whose structure is pictured in Figure 5.3; $\gamma$ is set equal to 1 and $\delta$ takes the values displayed in this figure.

A mesh generator gives an initial unstructured mesh. We only control the maximal diameter $h_{\max }$ of elements, and the generator takes the interfaces into account.

The matrix $B^{h}$ for the initial level is given by $\left(G^{h}\right)^{t} G^{h}$, the simplest choice underlined in Remark 4.6. The nodal prolongation matrix $\alpha$ is obtained from the solution of (3.1) with the bilinear form $c(\cdot, \cdot)$ associated with $\left(G^{h}\right)^{t} G^{h}$. The sets $L_{n}$ and the 


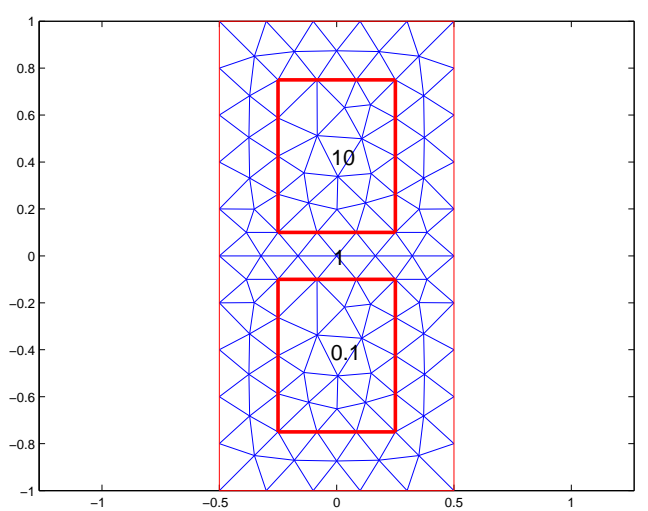

FIG. 5.3. Geometry of the problem with the values of $\delta$.

matrix $G^{H}$ are defined as in 4.35) and (4.37) with the map ind constructed from the aggregation algorithm given in [15].

5.4.1. Number of unknowns. In Table 5.7, the number of unknowns on each level is given for several choices of $h_{\max }$. The notation new denotes our algorithm, and RS denotes the algorithm of Reitzinger and Schöberl given in [14].

\begin{tabular}{c|c|c|c|c} 
Method & $h_{\max }=0.2$ & 0.1 & 0.05 & 0.025 \\
\hline new & $276+31$ & $1005+114+6$ & $3843+405+23$ & $15957+1467+82+3$ \\
\hline RS & $276+31$ & $1005+114+11$ & $3843+405+29$ & $15957+1467+151+21$ \\
\multicolumn{5}{c}{ TABLE 5.7} \\
Number of unknowns on each level for several choices of $h_{\max }$
\end{tabular}

\subsubsection{Computation of the Lagrange multipliers.}

Number of multipliers. The number of multipliers for every mesh and every level is given in Table 5.8. This number is roughly twice the number of unknowns for every

\begin{tabular}{c|c|c|c}
$h_{\max }=0.2$ & 0.1 & 0.05 & 0.025 \\
\hline 536 & $2105+179$ & $8049+759$ & $31249+2946+118$ \\
\multicolumn{4}{|c}{ TABLE 5.8 } \\
Number of Lagrange multipliers
\end{tabular}

level.

Stopping criteria on the residual. The Euclidean norm of the residual is divided by $10^{3}$. The number of iterations required to compute the Lagrange multipliers with different norms are gathered in Table 5.9. Compared to the results of Table 5.3, we can observe a significant increase for all methods but the results are similarly ordered.

5.4.3. Solution of system (2.4). The multilevel method is used as a preconditioner; the results for the different norms are gathered in Table 5.10 . 


\begin{tabular}{c|c|c|c|c} 
Norm & $h_{\max }=0.2$ & 0.1 & 0.05 & 0.025 \\
\hline$A$ & 387 & $\mathrm{X}+134$ & $\mathrm{X}+407$ & $\mathrm{X}+\mathrm{X}+136$ \\
\hline$A(\mathrm{GSsym})$ & 415 & $396+\mathrm{X}$ & $360+\mathrm{X}$ & $348+450+\mathrm{X}$ \\
\hline$A+G^{h} M_{\phi}^{-1}\left(G^{h}\right)^{t}$ & 62 & $103+31$ & $117+46$ & $120+59+26$ \\
\hline$A+G^{h} M_{\phi}^{-1}\left(G^{h}\right)^{t}(\mathrm{GSsym})$ & 94 & $128+37$ & $112+58$ & $113+59+27$ \\
\hline$S+\operatorname{reg}(0.1)$ & 74 & $212+32$ & $319+65$ & $326+200+27$ \\
\hline Id & 8 & $13+7$ & $13+12$ & $14+15+2$ \\
& TABLE 5.9 & & &
\end{tabular}

Number of iterations for the multiplier computation (division of the Euclidean norm of the residual by $10^{3}$ ) - unstructured meshes. X means that the stopping criterion was not reached after 1000 iterations.

\begin{tabular}{c|c|c|c|c} 
& $h_{\max }=0.2$ & 0.1 & 0.05 & 0.025 \\
\hline$A$ & 11 & 12 & 18 & 45 \\
\hline$A(\mathrm{GSsym})$ & 12 & 12 & 17 & 25 \\
\hline$A+G^{h} M_{\phi}^{-1}\left(G^{h}\right)^{t}$ & 12 & 13 & 17 & 26 \\
\hline$A+G^{h} M_{\phi}^{-1}\left(G^{h}\right)^{t}(\mathrm{GSsym})$ & 12 & 13 & 18 & 27 \\
\hline$S+\operatorname{reg}(0.1)$ & 12 & 12 & 16 & 25 \\
\hline Id & 13 & 13 & 18 & 28 \\
\hline R. S. & 14 & 20 & 33 & 56
\end{tabular}

Number of iterations of the conjugate gradient preconditioned by a multilevel method (division of the Euclidean norm of the residual by $\left.10^{10}\right)$.

The choices Id and $A+G^{h} M_{\phi}^{-1}\left(G^{h}\right)^{t}$ (GSsym) are the most efficient because they provide us with a performing preconditioner and the lowest computationnal cost for the setup phase.

The prolongation matrix $\beta$ built by our method provides us with a more efficient preconditioner than the method proposed by Reitzinger and Schöberl in 114. Thus in this simple example, the number of iterations approximately grows like $h_{\max }^{-0.75}$ for the method of Reitzinger and Schöberl and like $h_{\max }^{-0.5}$ for the choice Id. This validates the theoretical interest of our algebraic method.

However, the construction of the matrix $\beta$ remains really more expensive than in Reitzinger and Schöberl AMG method and the solution of the initial problem with our method is finally more time-consuming. In order to improve the efficacy for the solution of problem (3.11), an algorithm, which avoids the Lagrange multiplier computation, can be employed 12 .

\section{REFERENCES}

[1] D. N. Arnold, R. S. FAlk, And R. Winther, Multigrid in $\mathbb{H}($ div) and $\mathbb{H}$ (curl), Numer. Maths, 85 (2000), pp. 197-217.

[2] Pavel B. Bochev, C. Garasi, J. Hu, A. Robinson, and R. Tuminaro, An improved algebraic multigrid method for solving Maxwell's equations, SIAM J. Sci. Comput., 25 (2003), pp. 623-642 (electronic).

[3] Susanne C. Brenner and L. Ridgway Scott, The mathematical theory of finite element methods, vol. 15 of Texts in Applied Mathematics, Springer-Verlag, New York, second ed., 2002 . 
[4] Paul W. Gross and P. Robert Kotiuga, Electromagnetic theory and computation: a topological approach, vol. 48 of Mathematical Sciences Research Institute Publications, Cambridge University Press, Cambridge, 2004.

[5] R. Hiptmair, Multigrid method for Maxwell's equations, SIAM J. Numer. Anal., 36 (1999), pp. 204-225.

[6] J. Hu, R. Tuminaro, P. Bochev, C. Garasi, and A. Robinson, Toward an h-independent algebraic multigrid method for Maxwell's equations. To appear in SIAM J. Sci. Computing, 2005.

[7] M. Kaltenbacher and S. Reitzinger, Algebraic multigrid methods for nodal and edge based discretizations of Maxwell's equations, International Compumag Society Newsletter, 9 (2002), pp. 15-23.

[8] J. Mandel, M. Brezina, And P. VAnĚK, Energy optimization of algebraic multigrid bases, Computing, 62 (1999), pp. 205-228.

[9] T. Mifune, T. Inashita, and M. Shimasaki, A fast solver for fem analyses using the parallelized algebraic multigrid method, IEEE Trans. on Mag., 38 (2002), pp. 369-372.

[10] _ New algebraic multigrid preconditioning for iterative solvers in electromagnetic finite edge-element analyses, IEEE Trans. on Mag., 39 (2003), pp. 1677-1680.

[11] Peter Monk, Finite element methods for Maxwell's equations, Numerical Mathematics and Scientific Computation, Oxford University Press, New York, 2003.

[12] F. Musy, L. Nicolas, and R. Perrussel, Gradient-prolongation commutativity and graph theory, C. R. Acad. Sci. Paris, 341 (2005), pp. 707-712.

[13] J. C. NÉDÉLEC, Mixed finite elements in $\mathbb{R}^{3}$, Num. Math., 35 (1980), pp. 315 - 341.

[14] S. Reitzinger and J. SchöBerl, An algebraic multigrid method for finite element discretizations with edge elements, Numer. Linear Algebra Appl., 9 (2002), pp. 223-238.

[15] P. VAnĚK, J. Mandel, And M. BRezina, Algebraic multigrid by smoothed aggregation for second and fourth order elliptic problems, Computing, 56 (1996), pp. 179-196. International GAMM-Workshop on Multi-level Methods (Meisdorf, 1994).

[16] W. L. Wan, Tony F. Chan, And Barry Smith, An energy-minimizing interpolation for robust multigrid methods, SIAM J. Sci. Comput., 21 (1999/00), pp. 1632-1649 (electronic).

[17] K. Watanabe and H. Igarashi, On robustness of edge-based finite-element analysis using algebraic multigrid method, COMPEL, 24 (2005), pp. 408-417.

[18] K. Watanabe, H. Igarashi, and T. Honma, Comparison of geometric and algebraic multigrid methods in edge-based finite-element analysis, IEEE Trans. on Mag., 41 (2005), pp. 16721675 .

[19] Jinchao Xu and Ludmil Zikatanov, On an energy minimizing basis for algebraic multigrid methods, Comput. Vis. Sci., 7 (2004), pp. 121-127. 